\title{
Koordination von Raumplanung und Wasserbau als wesent- licher Bestandteil des Hochwasserrisikomanagements
}

\author{
Walter Seher · Clemens Neuhold
}

Angenommen: 20. Dezember 2021 / Online publiziert: 31. Januar 2022

(c) Der/die Autor(en) 2022

\begin{abstract}
Zusammenfassung Als Folge der Umsetzung der EU-Hochwasserrichtlinie haben flächen- und raumbezogene Aspekte im Hochwasserrisikomanagement an Bedeutung gewonnen. Dadurch und durch Herausforderungen wie die hohe Flächeninanspruchnahme für Bauland und Verkehrsflächen erhöht sich der Koordinationsbedarf zwischen Raumplanung und Wasserbau. Die ÖROK-Empfehlungen Nr. 57 „Hochwasserrisikomanagement" tragen diesem Umstand Rechnung und geben als Ergebnis einer inhaltlichen Abstimmung zwischen Raumplanungsund Wasserbauakteuren Leitlinien für den Umgang mit Hochwassergefahren und Hochwasserrisiken in der Raumplanung vor. Der vorliegende Beitrag nimmt diese ÖROK-Empfehlungen als Grundlage und legt den Schwerpunkt

der örtlichen Raumplanung ist teils in den Raumordnungsgesetzen mit Bezug $\mathrm{zu}$ bestimmten Grenzwerten der Gefährdung festgelegt, teils gewährleistet die administrative Koordination zwischen den Gemeinden und den wasserbaulichen Fachdiensten im Raumordnungsverfahren eine standardisierte Vorgehensweise bei der Gefahrenbeurteilung. Von großer Bedeutung ist die administrative Koordination von Raumplanung und Wasserbau auch im Umgang mit gewidmetem Bauland in Zonen mit mittlerer Überflutungswahrscheinlichkeit und bei pluvialen Hochwassergefahren sowie bei Nutzungsentscheidungen in Hochwasserabflussbereichen mit geringer Eintrittswahrscheinlichkeit und Restrisikogebieten, wo im Raumordnungsrecht nur vereinzelt normative Vorgaben bestehen.
\end{abstract} auf jene Empfehlungen, die direkt auf die Koordination der überörtlichen bzw. örtlichen Raumplanung mit dem Wasserbau abzielen. Im Zusammenhang mit der empfohlenen Berücksichtigung von Hochwasserabfluss- und Hochwasserrückhalteflächen in den Instrumenten der überörtlichen Raumplanung ist besonders die inhaltliche Abstimmung zwischen Raumplanung und Wasserbau gefordert, um die Freihaltung dieser Flächen als Anliegen der Regionalplanung in den Raumordnungsgesetzen $\mathrm{zu}$ verankern. Die Berücksichtigung von Gebieten mit Überflutung hoher und mittlerer Wahrscheinlichkeit in

\footnotetext{
Ass.-Prof. DI Dr. W. Seher $(\bowtie)$ Institut für Raumplanung, Umweltplanung und Bodenordnung, Universität für Bodenkultur Wien, Wien, Österreich walter.seher@boku.ac.at

\section{Dr. C. Neuhold}

Abteilung I/6 -

Hochwasserrisikomanagement,

Bundesministerium für

Landwirtschaft, Regionen und

Tourismus, Wien, Österreich

clemens.neuhold@bmlrt.gv.at
}

Schlüsselwörter Überörtliche und örtliche Raumplanung · Wasserbau . Politikkoordination .

Hochwasserrisikomanagement .

ÖROK-Empfehlungen

Coordination of spatial planning and hydraulic engineering as an essential part of flood risk management

Abstract Resulting from the implementation of the EU Floods Directive, spatial and land use issues have gained importance in flood risk management. This aspect and challenges such as the high land take for building and transport infrastructure increase coordination requirements between spatial planning and hydraulic engineering. The recommendations No. 57 of the Austrian Conference on Spatial Planning (OEROK) concerning flood risk management take this into account and provide guidelines for mitigating flood hazards and flood risks in spatial planning as a result of a substantive coordination between spatial planning and hydraulic engineering stakeholders. This contribution is based on these recommendations and focuses on recommendations directly aiming at the coordination of regional and local spatial planning with hydraulic engineering. In connection with the recommended consideration of flood runoff and flood retention areas in the instruments of regional land use planning, particularly substantive coordination between spatial planning and hydraulic engineering is required in order to include the preservation of these areas as a concern of regional planning into spatial planning laws. Considering areas of high and medium flooding probability in local land use planning is partly regulated in the spatial planning laws, partly administrative coordination between municipalities and hydraulic engineering authorities ensures a standardised approach to risk assessment in the planning process. Administrative coordination of spatial planning and hydraulic engineering is also of great importance when deciding on zoned building land in areas with medium flooding probability and in areas with pluvial flood hazards, as well as in land use decisions in runoff areas with low flooding probability and in residual risk areas, where regulation in spatial planning laws is widely lacking.

Keywords Spatial planning at regional and local level · Hydraulic engineering · Policy coordination . Flood risk management .

Recommendations of the Austrian Conference on Spatial Planning

\section{Einleitung: Die Rolle der Raumplanung im} Hochwasserrisikomanagement

Die Auswirkungen der Hochwasserereignisse der letzten Jahrzehnte haben einen Wandel im Umgang mit Hochwassergefahren eingeleitet. Anstelle der hauptsächlich auf technischen Schutzbauten basierenden Gefahrenabwehr wurden in den vergangenen Jahren zunehmend integrative Ansätze des 
Hochwasserrisikomanagements entwickelt, die ein Bündel an Schutz-, Vorsorge- sowie Bewältigungsmaßnahmen zur Verringerung von Hochwasserrisiken beinhalten (Nordbeck 2014). Einen maßgeblichen Treiber dieses Paradigmenwechsels stellt die EU-Hochwasserrichtlinie (Richtlinie 2007/60/EG über die Bewertung und das Management von Hochwasserrisiken) dar, mit der der integrierte Ansatz des Hochwasserrisikomanagements in Österreich verankert wurde.

Die Raumplanung ist Teil des Hochwasserrisikomanagements. Grundsätzlich besteht die Aufgabe der Raumplanung darin, Rahmenbedingungen für die planmäßige Gestaltung eines Gebiets zu setzen. Mit den Instrumenten der Raumplanung sollen Raumansprüche und -nutzungen entsprechend der Eignung der jeweiligen Standorte möglichst unter Vermeidung von Nutzungskonflikten zugeteilt werden. Die Hochwassergefährdung eines Standorts schränkt dessen Eignung für Wohnen, Gewerbe, Industrie, Freizeit und Verkehr erheblich ein. Nutzungen wie diese ziehen in Gefahrenbereichen ein je nach Art, Ausmaß und Eintrittswahrscheinlichkeit des Hochwasserereignisses ausgeprägtes Risiko nach sich.

Die Raumplanung ist den präventiven Maßnahmen im Hochwasserrisikomanagement zuzurechnen. Prävention durch Raumplanung beinhaltet in Abstimmung mit wasserbaulichen Schutzzielen (z.B. die $\mathrm{HQ}_{100}$-Überflutungsfläche oder Gefahrenzonen) einerseits die Festlegung akzeptabler Risiken und andererseits die Vermeidung neuer inakzeptabler Risiken durch Freihaltung von Gefahrengebieten von baulichen Intensivnutzungen (z.B. Siedlungsgebiete und relevante Infrastruktureinrichtungen) bzw. durch eine an die Gefahr angepasste Nutzung solcher Gebiete. Durch die Berücksichtigung der Risiken in der raumplanerischen Abwägung können Gefahren und Nutzungsinteressen für die Grundeigentümer verbindlich aufeinander abgestimmt werden. Dies geschieht einerseits in den Instrumenten der Raumplanung selbst, andererseits in den auf der Raumplanung aufbauenden Entscheidungen des Baurechts (Seher 2020a).

Die Festlegung von Schutzzielen auf Basis wasserbaulicher Überlegungen erfolgt in den Raumordnungsgesetzen der Bundesländer in Form von Bestimmungen zur Baulandeignung. Flächen eignen sich nicht als Bauland, wenn u.a. ungünstige natürliche Verhältnisse vorliegen, die durch technisch mögliche und wirtschaftlich vertretbare Maßnahmen nicht abgewendet werden können. Dazu zählen auch die Hochwassergefahren. Ist eine Fläche nicht als Bauland geeignet, darf sie im Flächenwidmungsplan auch nicht als Bauland gewidmet werden. Daraus resultieren Baulandwidmungsverbote in potenziellen $\mathrm{Ge}$ fahrengebieten bzw. Gefahrenzonen, die in den Raumordnungsgesetzen sowie in Instrumenten der überörtlichen Raumplanung normiert sind (Seher 2020a). Die Gefahrengebiete werden aktuell in den Raumordnungsgesetzen von Nieder- und Oberösterreich anhand von Abflussbereichen und Gefahrenzonen festgelegt, in den Raumordnungs- bzw. Raumplanungsgesetzen aller anderen Bundesländer werden die für die Bestimmung der Baulandeignung relevanten Gefahrengebiete nicht näher definiert. Eine Konkretisierung dieser unbestimmten Gefahrenbezeichnung in überörtlichen Raumordnungsprogrammen erfolgt gegenwärtig im Burgenland im Landesentwicklungsprogramm 2011 und in der Steiermark im landesweit gültigen Sachprogramm „zur hochwassersicheren Entwicklung der Siedlungsräume“.

Die Raumplanung ist sowohl was die planliche Darstellung von Gefahrenbereichen als auch die fachlichen Grundlagen für die Risikobewertung betrifft auf Informationen von naturgefahrenbezogenen Fachdiensten angewiesen. Für Hochwassergefahren sind das abhängig vom jeweiligen Gewässer die Bundeswasserbauverwaltung (BWV) mit den jeweils zuständigen Abteilungen für Wasserbau und wasserwirtschaftliche Planung in den Ämtern der Landesregierungen, die Wildbachund Lawinenverbauung (WLV, wie die BWV im Bundesministerium für Landwirtschaft, Regionen und Tourismus angesiedelt) sowie die Abteilung Bundeswasserstraßen im Bundesministerium für Klimaschutz, Umwelt, Energie, Mobilität, Innovation und Technologie. Diese Institutionen stellen verschiedene Informationsgrundlagen zur Beurteilung der Hochwassergefährdung zur Verfügung. Dazu zählen die Gefahrenzonenpläne der BWV und $\mathrm{WLV}$, die Abflussuntersuchungen der Bundeswasserbauverwaltung und der Bundeswasserstraßenverwaltung, die Hochwassergefahren- und -risikokarten gemäß EU-Hochwasserrichtlinie sowie die Gefahrenhinweiskarten für pluviale
Hochwasserereignisse. Über die Gefahreninformation hinaus sind die wasserbaulichen Fachdienste in der Gefahrenbeurteilung operativ tätig, besonders durch fachliche Stellungnahmen in Raumordnungs- und Bauverfahren auf kommunaler Ebene. Im Gegenzug spielen die Planungsträger der überörtlichen und örtlichen Raumplanung eine bedeutende Rolle in der Umsetzung raumplanungsrelevanter Maßnahmen, die im Hochwasserrisikomanagementplan gemäß EU-Hochwasserrichtlinie festgelegt sind. Diese Interaktionsfelder zwischen Wasserbau und Raumplanung erfordern Koordination im Sinne einer Definition von Schnittstellen und einer Gestaltung von formellen und informellen Abstimmungsmechanismen, stellen also die formale Ebene der Koordination zwischen Raumplanung und Hochwasserrisikomanagement dar.

Auf inhaltlicher Ebene stellt besonders die Überlagerung von potenziellen Siedlungsgebieten mit einerseits Gefahrengebieten und andererseits jenen Flächen, die für Hochwasserabfluss und Hochwasserrückhalt benötigt werden, eine Herausforderung für die Koordination zwischen Raumplanung und Hochwasserrisikomanagement dar. Trotz Fortschritten bei der Eindämmung des Anstiegs der Hochwasserexposition von Menschen und Sachwerten bleibt die Siedlungsentwicklung ein wesentlicher Treiber des Hochwasserrisikos (Elmer et al. 2012; Löschner et al. 2017). Hohe Risiken treten verstärkt in baulich intensiv genutzten Gebieten gegenüber Szenarien mittlerer bzw. geringer Gefährdung auf, insbesondere auch in jenen Bereichen, in denen technische Schutzmaßnahmen eine intensivere Landnutzung wie eine Bebauung auf ehemals gefährdeten Flächen ermöglichen (Nachtnebel und Apperl 2015). Diese potenziellen Risikogebiete werden im Allgemeinen von den bestehenden Restriktionen betreffend Baulandwidmung in Gefahrenbereichen nicht erfasst. Eine Ausnahme bildet das Oberösterreichische Raumordnungsgesetz, wo festgelegt wird, dass selbst rote Gefahrenzonen, die vor Umsetzung von wasserbaulichen Maßnahmen ausgewiesen wurden, auch nach der Umsetzung von Maßnahmen von einer Baulandwidmung freizuhalten sind (ehemals rote Zonen). Zum Anstieg des Hochwasserrisikos trägt in Österreich auch die hohe Flächeninanspruchnahme für Bauland und Verkehrsflächen bei (Umweltbundes- 
amt 2021; BMLRT 2021), die besonders in den Talbereichen entlang der größeren Flüsse intensiver ausgeprägt ist (Seher 2015). Die Flächeninanspruchnahme erhöht nicht nur das Hochwasserrisiko, sondern führt auch zu einem weiteren Verlust von Hochwasserabfluss- und Hochwasserrückhalteflächen und konterkariert damit Bemühungen der nationalen und europäischen Hochwasserpolitik, mehr „Raum für die Flüsse" (Warner et al. 2012) zu schaffen. Übergeordnetes Ziel einer Koordination von Raumplanung und Hochwasserrisikomanagement sollte es - besonders vor dem Hintergrund von möglichen Einflüssen des Klimawandels auf das Hochwassergeschehen - sein, neue Hochwasserrisiken trotz des Drucks in Richtung weiterer Siedlungsentwicklung zu vermeiden und geeignete Flächen für den Hochwasserabfluss und Hochwasserrückhalt von Bebauung freizuhalten.

Vor dem Hintergrund dieser formalen und sachlichen Anforderungen beschäftigt sich dieser Beitrag mit der inhaltlichen und administrativen Koordination von Raumplanung und Wasserbau im Rahmen der ÖREK-Partnerschaft ${ }^{1}$ „Risikomanagement Hochwasser". Als Resultat dieser Partnerschaft wurden zehn Empfehlungen zum Hochwasserrisikomanagement erarbeitet und zusammen mit Erläuterungen und die Empfehlungen illustrierenden Beispielen als ÖROKEmpfehlung Nr. 57 „Hochwasserrisikomanagement" veröffentlicht (ÖROK 2018). Nach einer kurzen Vorstellung dieser ÖREK-Partnerschaft wird der Schwerpunkt der weiteren Ausführungen auf jene Empfehlungen gelegt, die sich explizit mit der Abstimmung von Wasserbau und überörtlicher bzw. Wasserbau und örtlicher Raumplanung befassen.

Dieser Beitrag basiert vorrangig auf den Empfehlungstexten und den Erläuterungen zur ÖROK-Empfehlung Nr. 57. Zusätzlich fließen Ergebnisse einer Serie von Experteninterviews mit Stakeholdern aus den Bereichen Raumplanung und Wasserbau, die im Zuge des von der Österreichischen Akademie der Wissenschaften geförderten Forschungsprojekts PoCo-FLOOD (Integrales Hochwasserrisikomanagement

\footnotetext{
1 Umsetzungspartnerschaft zum Österreichischen Raumentwicklungskonzept (ÖREK) 2011.
}

in Berggebieten: sektorale Interdependenzen, Konflikte und Möglichkeiten der Politikkoordination ${ }^{2}$ ) u. a. zum Thema Koordination von Raumplanung und Wasserbau geführt wurden, in diesen Beitrag mit ein.

\section{Die ÖREK-Partnerschaft} „Risikomanagement Hochwasser" und die ÖROKEmpfehlungen Nr. 57 „Hochwasserrisikomanagement"

Um die Umsetzung des Österreichischen Raumentwicklungskonzepts (ÖREK) 2011 zu unterstützen, wurden themenbezogene „ÖREK-Partnerschaften" ins Leben gerufen. In diesen Partnerschaften setzten sich ÖROKMitglieder und weitere relevante Akteure in Projektarbeitsgruppen mit der Umsetzung von Aufgaben aus dem jeweiligen Themenbereich auseinander. Die ÖREK-Partnerschaft „Risikomanagement Hochwasser" unter Federführung des damaligen Bundesministeriums für Nachhaltigkeit und Tourismus (BMNT), (damalige) Abteilung Schutzwasserwirtschaft, und des Landes Salzburg, Referat Raumplanung, setzte sich u.a. zum Ziel, wesentliche Themenfelder der ÖROK-Empfehlung Nr. 52 (zu Raumordnung und Naturgefahren) neu zu diskutieren und vom präventiven Ansatz des Hochwasserschutzes in Richtung eines integralen Risikomanagements weiter $\mathrm{zu}$ entwickeln. ÖROK-Empfehlungen werden zu relevanten Fragen der Raumordnung und Regionalpolitik erstellt und richten sich in erster Linie an die ÖROKMitglieder. Auf Basis von wissenschaftlichen Grundlagenarbeiten und einer intensiven Abstimmung unter den beteiligten Akteuren werden gemeinsam erarbeitete und politisch akkordierte Handlungsempfehlungen formuliert (ÖROK 2018).

Seit der Erstellung der ÖROK-Empfehlung Nr. 52 wurden zahlreiche Aktivitäten im Bereich Hochwasserrisikomanagement gesetzt. Zusätzlich brach-

${ }^{2}$ Im Original: Integrated Flood Risk Management in Mountain Areas: Assessing Sectoral Interdependencies, Conflicts and Options for Policy Coordination, vgl.https://www.researchgate.net/project/ PoCo-FLOOD-Integrated-Flood-RiskManagement-in-Mountain-AreasAssessing-Sectoral-InterdependenciesConflicts-and-Options-for-PolicyCoordination. ten geänderte Rahmenbedingungen auf EU-, Bundes- und Landesebene neue Anforderungen und Herausforderungen an die Planungsverantwortlichen mit sich. So stellt die EU-Hochwasserrichtlinie eine neue Planungsgrundlage dar. Auf nationaler Ebene bildet der „Nationale Hochwasserrisikomanagementplan 2015“ als Umsetzung dieser Richtlinie einen Rahmen, der für den Zeitraum 2016 bis 2021 (und darauffolgende 6-Jahres-Zyklen) angemessene Ziele zur Risikoreduktion definiert und Maßnahmen sowie deren Priorität zur Erreichung dieser Ziele umfasst. Vor diesem Hintergrund wurden die zehn Empfehlungen zu raumplanerisch relevanten Aspekten des Hochwasserrisikomanagements formuliert. Unter dem Handlungsgrundsatz „dem Wasser Raum geben" behandeln die einzelnen Empfehlungen unter anderem die Vermeidung nachteiliger Folgen für die menschliche Gesundheit, die Umwelt, das Kulturerbe, die wirtschaftliche Tätigkeit und die räumliche Entwicklung sowie die Freihaltung von gefährdeten Flächen, insbesondere für den Hochwasserabfluss und -rückhalt und für die Gewässerentwicklung (ÖROK 2018).

Trein et al. (2021) unterscheiden zwischen den Kategorien „administrative Koordination“ und „Politikintegration.“ Administrative Koordination meint die Koordination von Organisationen des öffentlichen Sektors. Die administrative Koordination beruht auf der Anwendung von Verfahrensinstrumenten, die auf die Zusammenarbeit von Akteuren aus verschiedenen öffentlichen Politikbereichen abzielen. Politikintegration oder inhaltliche Koordination spricht die Verknüpfung öffentlicher Politikbereiche an. Die ÖROK-Empfehlungen zum Hochwasserrisikomanagement sind der Politikintegration zuzurechnen. Die folgenden Ausführungen beschäftigen sich auf Grundlage ausgewählter ÖROK-Empfehlungen mit der Interaktion von inhaltlicher und administrativer Koordination zwischen Raumplanung und Wasserbau.

\section{Koordination Wasserbau - überörtliche Raumplanung}

Die Empfehlungen 3 und 4 der ÖREKPartnerschaft „Risikomanagement Hochwasser" sprechen die Sicherung von Flächen für den Hochwasserabfluss und den Hochwasserrückhalt sowie zur Gewässerbewirtschaftung auf überörtlicher Ebene an und verweisen auf die 
Abstimmung zwischen Wasserbau und überörtlicher Raumplanung. Dabei soll die Wasserwirtschaft strategisch ausgerichtete regionale Planungsinstrumente zur Verfügung stellen und die notwendigen Flächen vorrangig mittels überörtlicher Raumplanungsinstrumente oder wasserwirtschaftlicher Regionalprogramme gesichert werden (ÖROK 2018).

Die Erhaltung des natürlichen Wasserrückhalts und die Sicherung von Hochwasserabflussräumen sowie die Verringerung von Hochwasserrisiken sind zentrale Themenstellungen eines integrierten Hochwasserrisikomanagements. Die EU-Hochwasserrichtlinie sieht ein integrativ ausgerichtetes Hochwasserrisikomanagement auf Einzugs- oder Flussgebietsebene vor und forciert die Erhaltung und Wiederherstellung von Überflutungsflächen. Dem gegenüber steht die Tatsache, dass eine im gegenwärtigen Ausmaß weiter fortschreitende Flächeninanspruchnahme für Bauland und Verkehrsflächen zwangsläufig zu einer weiteren Reduktion geeigneter Überflutungsflächen führen wird (vgl. Kap. 1).

Vor diesem Hintergrund ist ein zielgerichteter Umgang mit Überflutungsflächen erforderlich, der sowohl methodische Grundlagen zur quantitativen Beurteilung der natürlichen Rückhalteund Abflusswirkung und strategische Planungsinstrumente als Grundlage der Flächensicherung als auch operativ wirksame Instrumente der Flächenfreihaltung selbst inkludiert.

\subsection{Strategische Planungsinstrumente des Wasserbaus}

Strategische Planungsinstrumente der Wasserwirtschaft stehen in Form von für bestimmte Flussabschnitte bzw. Einzugsgebiete vorliegenden Gewässerentwicklungskonzepten, Regionalstudien, Gewässerbetreuungskonzepten sowie Schutzwasserwirtschaftlichen Grundsatzkonzepten zur Verfügung. Mit dem Gewässerentwicklungs- und Risikomanagementkonzept (GE-RM, siehe auch Wenk und Mühlmann 2022, in diesem Heft) wurde ein Planungsinstrument der Wasserwirtschaft etabliert, das Synergien zwischen Hochwasserrisikomanagement und Gewässerökologie herstellen kann und die vorabgenannten Planungsinstrumente ablöst. Das Gewässerentwicklungs- und Risikomanagementkonzept ist eine zeitlich, sachlich und räum- lich übergeordnete flussraumbezogene Fachplanung, mit deren Hilfe die Planungen und Aktivitäten im Flussraum, insbesondere die Maßnahmen des Hochwasserrisikomanagements, mit den fachlich notwendigen und möglichen Maßnahmen für die Zielerreichung nach Wasserrahmenrichtlinie abgestimmt und vernetzt werden können. Gewässerentwicklungs- und Risikomanagementkonzepte legen mittel- bis langfristige Entwicklungsziele, Maßnahmenschwerpunkte und Handlungsoptionen für den Hochwasserschutz, die künftige Gewässerentwicklung, den Feststoffhaushalt und das Hochwasserrisikomanagement fest. Neben der Vernetzung und Abstimmung der wasserbaulichen und gewässerökologischen Planungen im Einzugsgebiet bzw. über längere Gewässerabschnitte, ist die Einbeziehung von anderen Planungsträgern (u.a. Raumplanung, Infrastrukturplanung, land- und forstwirtschaftliche Nutzung) ein wesentliches Merkmal dieses strategisch ausgerichteten integrativen Planungsinstruments (BMLFUW 2016). Die Ausweisung von für den Hochwasserabfluss und den Hochwasserrückhalt wesentlichen Flächen erfolgt in der Gefahrenzonenplanung mittels der rot-gelb schraffierten Funktionsbereiche (Schmid et al. 2022, in diesem Heft).

Als operative Instrumente der Flächensicherung eignen sich die Instrumente der überörtlichen Raumplanung und das Wasserwirtschaftliche Regionalprogramm (Vogl 2022, in diesem Heft).

\subsection{Planungsinstrumente der überörtlichen Raumplanung}

Entgegen der einzugs- und flussgebietsbezogenen Ausrichtung der strategischen Planungsinstrumente der Wasserwirtschaft liegt in Österreich der Schwerpunkt raumplanerischer Maßnahmen zur Vermeidung von Hochwasserrisiken gegenwärtig auf der örtlichen Planungsebene. Informationen zur Hochwassergefährdung und zum Hochwasserrisiko stehen zwar in regionalem Maßstab zur Verfügung, in der überörtlichen Raumplanung sind aber flächenbezogene Festlegungen zur Freihaltung von Hochwasserabflussund Hochwasserrückhalteflächen mit verpflichtendem Charakter nur vereinzelt vorgesehen. Die für den Umgang mit dem zumeist gemeindeübergreifenden Phänomen Flusshochwasser prä- destinierte Regionalplanung, mit den Regionalen Sachprogrammen und den Regionalen Raumordnungs- bzw. Entwicklungsprogrammen als Planungsinstrumenten, kennt in Österreich nur vereinzelt gesetzliche Verpflichtungen für Festlegungen bezüglich Naturgefahren, wie z.B. hochwasserbezogene Vorrang- oder Vorbehaltsflächen (Seher und Löschner 2018). Solche Festlegungen sind zwar nicht ausgeschlossen, zählen aber nicht zu den Kerninhalten der regionalen Raumplanung, weder was überörtliche Bauverbote noch was die Ersichtlichmachung von Gefahrenbereichen betrifft (Kanonier 2005). Auch ist die Regionalplanungsdichte - d.h. die flächige Abdeckung mit Regionalen Raumordnungsprogrammen österreichweit gering, wobei zwischen den einzelnen Bundesländern Unterschiede bestehen.

Dieser Mangel an hochwasserrelevanten Regelungen in der Regionalplanung wiegt deshalb schwer, weil nicht alle Bauführungen, die in Hochwasserabfluss- und Hochwasserrückhalteflächen geplant sind, von den Baulandwidmungsbeschränkungen in den Raumordnungsgesetzen erfasst werden: Zum einen gehen diese Flächen über die planungsrelevanten Gefahrenbereiche hinaus, zum anderen können Bauführungen auch außerhalb des gewidmeten Baulands getätigt werden (z. B. landwirtschaftliche Bauten im Grünland). Die schon angesprochene hohe Flächeninanspruchnahme für Bauland und Verkehrsflächen resultiert aus einer Vielzahl von Widmungsentscheidungen in den Gemeinden, die auf regionaler Ebene zu unerwünschten Folgewirkungen, wie u.a. zu einem schleichenden Verlust von Hochwasserabflussflächen, führen (Dallhammer 2006). Auch dieser Zusammenhang unterstreicht die Bedeutung der Regionalplanung, die einen wichtigen Beitrag zur Freihaltung großflächiger Hochwasserabflussflächen auf Flussgebietsebene leisten kann (Heiland 2002).

In Vorarlberg wurde „zur Verbesserung der räumlichen Voraussetzungen für den Hochwasserschutz" das Regionale Sachprogramm „Blauzone Rheintal" mit dem Ziel der Sicherung von Hochwasserabfluss- und wasserwirtschaftlichen Bedarfsflächen verordnet (Abb. 1). Diese überörtlichen Freiflächen („Blaue Zonen“) sind in den Flächenwidmungsplänen der betroffenen Gemeinden als Freifläche-Freihaltegebiet auszuweisen (Amt der Vorarlberger 


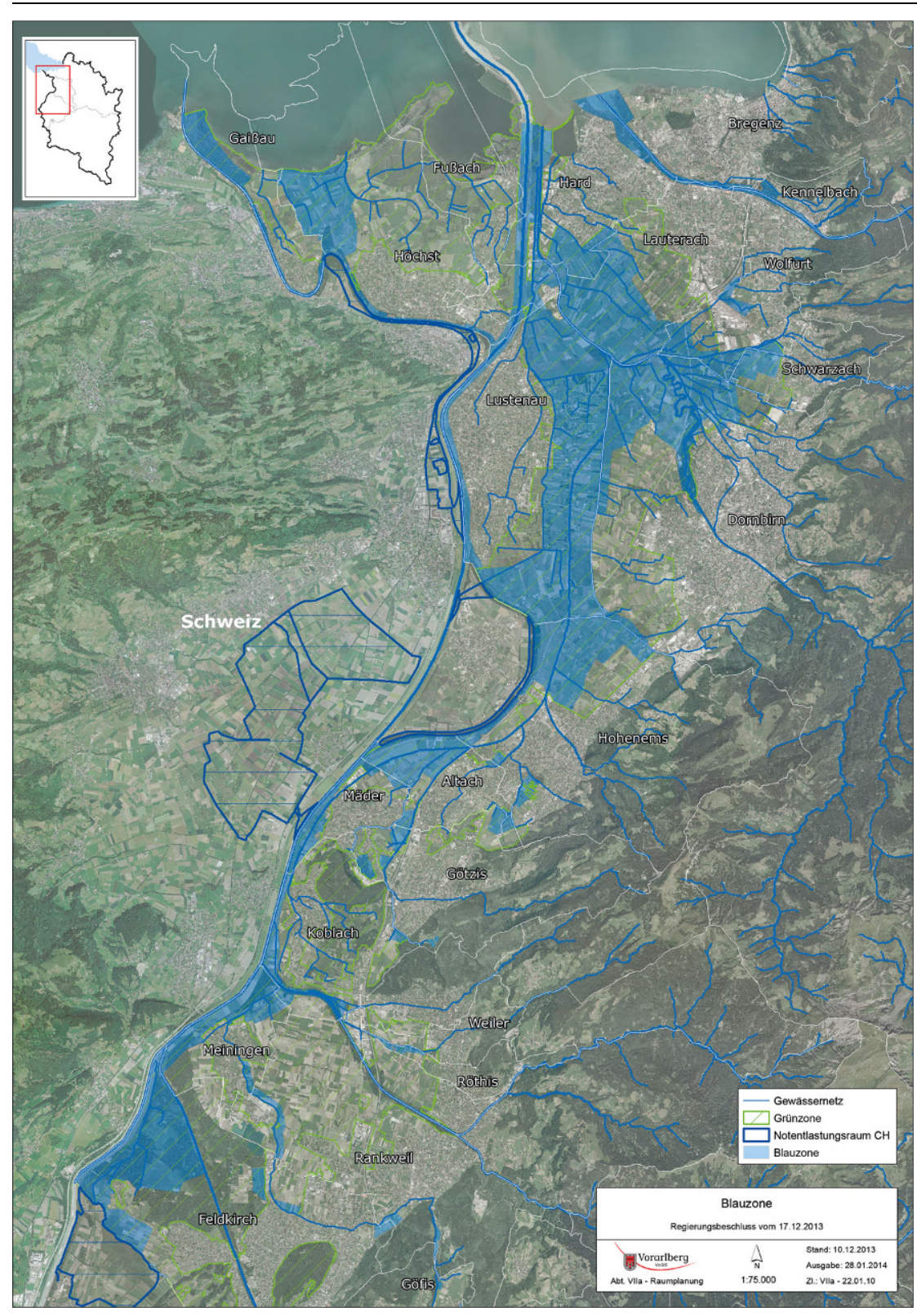

Abb. 1 Blauzone Rheintal (Amt der Vorarlberger Landesregierung 2014)

Landesregierung 2013). Damit können Flächen für den Hochwasserabfluss und Hochwasserrückhalt sowie für zukünftige wasserbauliche Maßnahmen erhalten und gesichert werden. Für die Abgrenzung der Blauzone wurden die wasserbaulichen Unterlagen als Planungsgrundlage mit raumplanungsfachlichen Kriterien abgestimmt. Kern dieser Grundlagendaten sind die Überflutungsberechnungen für ein 100- bzw. 300-jährliches Hochwasserszenario. Das Regionale Sachprogramm Blauzone Rheintal beinhaltet nicht nur die in Empfehlung 4 der ÖREK-Partnerschaft „Risikomanagement Hochwasser“ an- serbauverwaltung in das Programm zu integrieren. Ein Entwurf für ein Sachprogramm zu Raumplanung und Naturgefahren liegt auch in Kärnten vor, das im Sinne eines überörtlichen Zugangs konkretere Vorgaben für die örtliche Raumplanung festlegt.

Aktuell laufen in einigen Bundesländern Aktivitäten, die in Richtung einer Stärkung der überörtlichen Raumplanung, insbesondere der Regionalplanung gehen (ÖROK 2021a). Im Sinne der Abstimmung von Wasserbau und überörtlicher Raumplanung sollten wie in der Empfehlung zur überörtlichen Sicherung von Hochwasserabfluss- und Hochwasserrückhalteflächen vorgeschlagen regionale Freihaltezonen auch in diese Raumordnungsprogramme integriert werden.

\subsection{Wasserwirtschaftliches Regionalprogramm}

Das Wasserwirtschaftliche Regionalprogramm ist ein Instrument der wasserwirtschaftlichen Planung. Es stellt eine Weiterentwicklung der Wasserwirtschaftlichen Rahmenverfügung dar, die im Zuge der Umsetzung der EUHochwasserrichtlinie im Österreichischen Wasserrechtsgesetz inhaltlich um die Belange des Hochwasserrisikomanagements erweitert wurde. Wasserwirtschaftliche Regionalprogramme eröffnen u. a. die Möglichkeit, eine Widmung für bestimmte wasserwirtschaftliche Zwecke vorzunehmen oder die wasserrechtliche Bewilligungspflicht für die Errichtung und Änderung von Bauten im Abflussbereich des 30-jährlichen Hochwassers ( $\$ 38$ WRG) auf weiträumigere Hochwasserabflussbereiche (z. B. HQ100) auszudehnen. Wasserwirtschaftliche Regionalprogramme werden auf Grundlage der Gefahrenzonenplanung erstellt und vom jeweiligen Landeshauptmann als Verordnung erlassen. Sind mehrere Bundesländer erfasst, wie zum Beispiel bei der Betrachtung von gesamten Einzugsgebieten, wird die Verordnung von der Bundesministerin für Landwirtschaft, Regionen und Tourismus erlassen. Wasserwirtschaftliche Regionalprogramme eignen sich aufgrund ihres flexibel festlegbaren Planungsraums gut für eine fluss- oder einzugsgebietsbezogene Planung.

In Niederösterreich wurden für $11 \mathrm{Ge}$ wässerstrecken sogenannte Hochwasserschutzzonenpläne als Wasserwirtschaftliche Regionalprogramme verordnet. Die Hochwasserschutzzonenpläne 
weisen Überflutungsflächen bis zu einem 100-jährlichen Hochwasserereignis aus, die einen maßgeblichen Beitrag zum Hochwasserrückhalt leisten. Für diese Überflutungsflächen werden Regelungen für (bauliche) Vorhaben (insbesondere Aufschüttungen, Bauführungen im Grünland), die zu einem Verlust von Retentionsflächen führen können, festgelegt. Die Hochwasserschutzzonenpläne erfassen auch Summationseffekte. Die Vorgaben der Verordnungen beziehen sich ausschließlich auf die Bewertung des Hochwasserrückhalts (Amt der Niederösterreichischen Landesregierung o.J.). Diese flächenbezogenen Festlegungen ergänzen die in Niederösterreich bestehenden raumordnungsrechtlichen Festlegungen für den Abflussbereich des hundertjährlichen Hochwassers.

Mit dem Wasserwirtschaftlichen Regionalprogramm liegt ein nicht nur vom Namen her raumplanungsähnliches Planungsinstrument der Wasserwirtschaft vor. Auch die im WRG vorgesehene Möglichkeit, Flächen für bestimmte wasserwirtschaftliche Zwecke zu widmen, deutet auf einen Abstimmungsbedarf zwischen den Festlegungen von Wasserwirtschaftlichen Regionalprogrammen und den Planungsinstrumenten der überörtlichen und der örtlichen Raumplanung hin. Durch eine entsprechende Koordination sollte sichergestellt werden, dass Regelungen in Wasserwirtschaftlichen Regionalprogrammen jene räumlichen und inhaltlichen Bereiche betreffen, auf die die Raumplanung keinen Einfluss nehmen kann.

\section{Koordination Wasserbau - örtliche Raumplanung}

Empfehlung 6 der ÖREK-Partnerschaft „Risikomanagement Hochwasser" hat eine verpflichtende Verankerung von Gefahrenzonenplanungen und Abflussuntersuchungen in der örtlichen Raumplanung und im Baurecht ${ }^{3}$ zum Inhalt. Gebiete, die eine hohe und mittlere Hochwassergefährdung aufweisen, insbesondere rote Gefahrenzonen, sind grundsätzlich weder zu bebauen noch mit einer Widmung zu belegen, die eine Bauführung zulässt. Widmungsbeschränkungen in den Raumordnungs-

${ }^{3}$ Die weiteren Ausführungen in diesem Kapitel beziehen sich nur mehr auf die Raumplanung. gesetzen sollen an die Ergebnisse der Gefahrenzonenplanung und der Abflussuntersuchungen gekoppelt werden. Damit ist gemeint, dass in der Raumordnungsgesetzgebung ein expliziter Bezug dieser Widmungsbeschränkungen $\mathrm{zu}$ den im Wasserrechtsgesetz festgelegten Hochwassergefährdungsbereichen hergestellt werden soll (ÖROK 2018). Das Wasserrechtsgesetz definiert ein Hochwasser mit hoher Wahrscheinlichkeit als 30-jährliches Hochwasser, ein Hochwasser mit mittlerer Wahrscheinlichkeit zumindest als 100-jährliches Hochwasser sowie ein Hochwasser mit niedriger Wahrscheinlichkeit als 300-jährliches Hochwasser oder mittels Szenarien für Extremereignisse (vgl. § 55k Abs. 2 WRG).

\subsection{Rechtliche Rahmenbedingungen für die Flächenwidmung}

Ein derartiger Bezug ist gegenwärtig in den Raumordnungsgesetzen von Nieder- und Oberösterreich, im Landesentwicklungsprogramm des Burgenlands sowie im Steiermärkischen Sachprogramm „zur hochwassersicheren Entwicklung der Siedlungsräume“ gegeben (vgl. Kap. 1). Das Tiroler Raumordnungsgesetz stellt den Bezug zur Gefahrenzonenplanung dahingehend her, dass bei der Beurteilung der Baulandeignung auf Gefahrenzonenpläne Bedacht zu nehmen ist. In den anderen Bundesländern sind die hochwasserbezogenen Bestimmungen zur Baulandeignung unbestimmt formuliert. Die Intention dieser Empfehlung besteht auch darin, unbestimmte Bezeichnungen der Hochwassergefährdungsbereiche in den Raumordnungsgesetzen $\mathrm{zu}$ vermeiden und damit v.a. in der örtlichen Raumplanung anwendbare Festlegungen zu treffen.

Werden Gefahrenbereiche im jeweiligen Raumordnungsgesetz oder in überörtlichen Raumordnungsprogrammen nicht explizit benannt, ist im Raumordnungsverfahren eine Stellungnahme des zuständigen wasserbaulichen Fachdienstes erforderlich, die für Widmungsansuchen in der roten Gefahrenzonen in aller Regel negativ ausfällt. Für Widmungsansuchen in der gelben Gefahrenzone wird die Stellungnahme zur Beurteilung der Baulandeignung oft von Kriterien wie der Wassertiefe und der Fließgeschwindigkeit abhängig gemacht, und es werden gegebenenfalls bauliche Auflagen zur Hochwasseranpassung erteilt. Diese einzelfallbezogene Gefahrenbeurteilung steht für eine direkte Form der administrativen Koordination zwischen Wasserbau und örtlicher Raumplanung, die allerdings erhebliche zeitliche und personelle Ressourcen der zuständigen Behörden in Anspruch nimmt. Eine genaue Bezeichnung der Widmungsbeschränkung durch Grenzwerte der Gefährdung (Jährlichkeiten oder Gefahrenzonen) ermöglicht die Beurteilung der Baulandeignung durch Ortsplaner oder Gemeindeorgane, was zwar die Fachdienste entlastet, aber die Qualität der Gefahrenbeurteilung in jenen Bereichen vermindert, für die kein Baulandwidmungsverbot besteht. Für die Gefahrenbeurteilung von Bauführungen im Grünland (z.B. PhotovoltaikFreiflächenanlagen) oder auf Sonderflächen erscheint eine fachliche Stellungnahme des Wasserbaus jedenfalls weiter erforderlich.

\subsection{Berücksichtigung von Hochwassergefahren im Örtlichen Entwicklungskonzept}

Die Abgrenzung von hochwassergefährdeten Gebieten und Gebieten, die für eine Baulandentwicklung in Frage kommen, empfiehlt sich aber bereits für das Örtliche Entwicklungskonzept $(\text { ÖEK })^{4}$. In fast allen Bundesländern ist das ÖEK mittlerweile verbindlich zu erstellen. Auf der Grundlage von Gefahrenzonenplänen, Abflussuntersuchungen und Gefahrenhinweiskarten (für pluviale Hochwasserereignisse) kann die Baulandeignung schon vor der eigentlichen Widmung geklärt werden. Neben Ausschluss- und Eignungszonen werden im ÖEK auch Flächen mit Vorbehalt betreffend Naturgefahren ausgewiesen, die dann für eine Baulandwidmung eine Stellungnahme des Wasserbaus erfordern, die in weiterer Folge $\mathrm{zu}$ baulichen Auflagen im Bebauungsplan oder im Bauverfahren führt. Die Zonierung im ÖEK schafft damit bereits vorab Klarheit über jene Flächen, die mit oder ohne bauliche Auflagen für eine Baulandentwicklung in Frage kommen. Eine parzellenscharfe Berücksichtigung von Naturgefahren

${ }^{4}$ Die diesbezüglichen Bezeichnungen in den Bundesländern sind unterschiedlich. Dieses strategisch ausgerichtete Planungsinstrument der örtlichen Raumplanung wird auch als Räumliches Entwicklungskonzept oder als Räumlicher Entwicklungsplan bezeichnet. 
im Allgemeinen erfolgt erst im Flächenwidmungs- und gegebenenfalls im Bebauungsplan. Diese Vorgehensweise verdeutlicht, dass der Umgang mit Hochwassergefahren im ÖEK eine enge Abstimmung von Wasserbau und örtlicher Raumplanung erfordert. In die Erstellung eines ÖEK bringen sich verschiedene Fachbereiche ein, wodurch im Planungsprozess eines ÖEK der vorausschauenden Koordination unterschiedlicher Nutzungsansprüche in Form einer administrativen Koordination der Planungsträger eine besondere Bedeutung zukommt.

\subsection{Umgang mit gewidmetem Bauland in Gefährdungsbereichen}

In Österreich sind mit Stand 2020 bei unterschiedlicher regionaler Verteilung $22 \%$ des gewidmeten Baulands nicht bebaut (ÖROK 2021b). Diese Baulandreserven beinhalten in der Regel siedlungsstrukturell gut geeignetes Bauland und stellen damit neben den Gebäudeleerständen eine wichtige Ressource für die Innenentwicklung dar, die als Maßnahme zur Reduktion des „Flächenverbrauchs“ forciert wird ${ }^{5}$. Der Umgang mit unbebautem Bauland in hochwassergefährdeten Bereichen (Abb. 2) gestaltet sich damit als Interessenabwägung zwischen der Innenentwicklung und der Freihaltung gefährdeter Flächen. Diese Abwägung wird sich in den Gefährdungsbereichen, die durch pluviales Hochwasser hervorgerufen werden, häufiger stellen als in den $\mathrm{Ab}$ flussgebieten fluvialer Hochwasser. Das liegt einerseits an der - verglichen mit Flusshochwasser - höheren Exposition des Siedlungsbestands gegenüber pluvialem Hochwasser und andererseits an der besseren Beherrschbarkeit der Gefahrenprozesse durch kleinere bauliche Anpassungen. Bei entsprechender Baulandnachfrage in einer Gemeinde müssen Baulücken (gewidmetes Bauland), die im Siedlungsbereich nicht geschlossen werden, im Außenbereich gewidmet werden. So kann es unter Umständen sinnvoller sein, strukturelle Schutzmaßnahmen zu setzen und Baulücken in Innerortslagen zu bebauen, auch im Hinblick auf die finanziellen Vorleistungen, die die Gemeinde

${ }^{5}$ Zum Anteil jener Baulandreserven, die in von (fluvialem und pluvialem) Hochwasser gefährdeten Gebieten liegen, liegen keine belastbaren Zahlen vor.

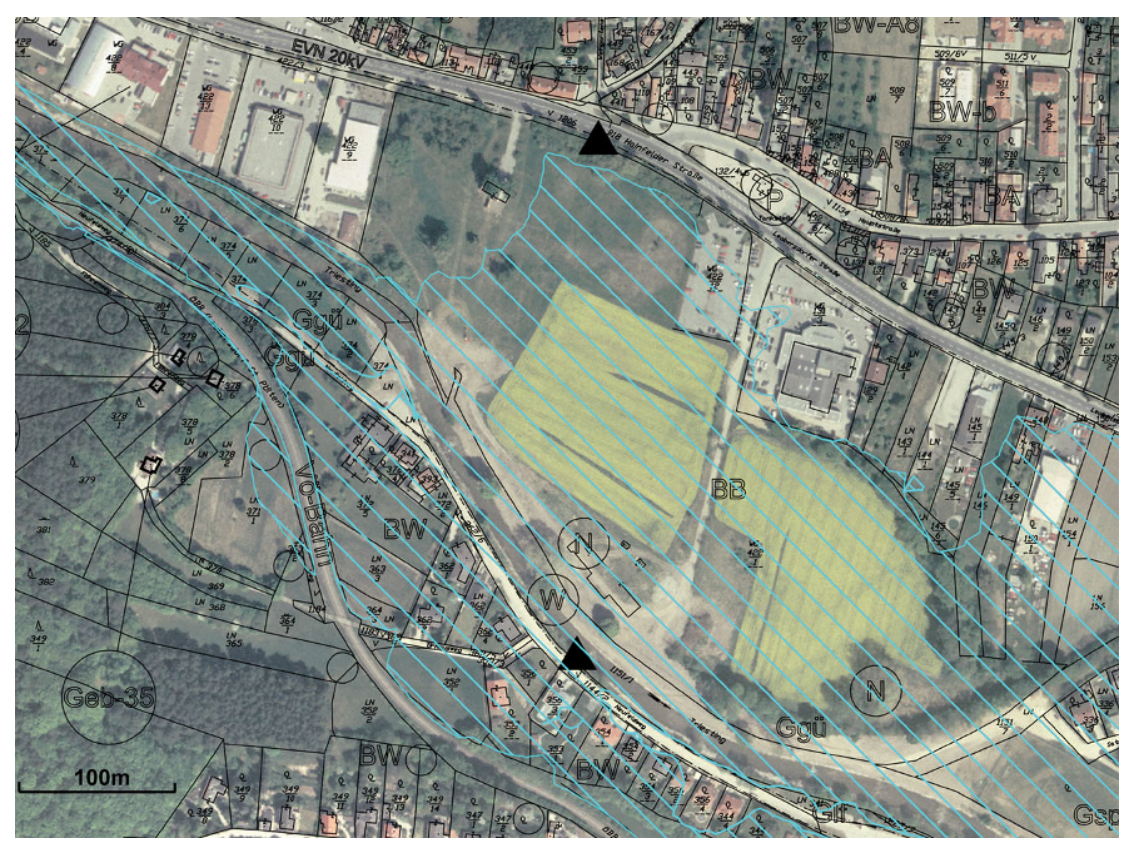

Abb. 2 Gewidmetes Bauland im Hochwasserabflussbereich (Quelle: Seher und Beutl 2004)

schon zur Erschließung des Baulands getätigt hat (Seher 2020b).

Diese Abwägung im Raumordnungsverfahren muss auf fachlichen Einschätzungen des Wasserbaus basieren, was eine entsprechende Abstimmung erfordert. In Abhängigkeit von der Gefährdungslage und der Bedeutung der Fläche für den Oberflächenabfluss werden die zuständigen Fachdienste bauliche Anpassungsmaßnahmen festlegen, die im Zuge des Bauverfahrens umzusetzen sind, oder eine Bebauung der Fläche ausschließen. Besteht die Option, die Gefährdung der Fläche durch technische Schutzmaßnahmen zu reduzieren, sind Bausperren oder die Widmung von Aufschließungszonen geeignete Planungsinstrumente, die eine Bebauung bis zur Herstellung der Baulandeignung unterbinden. Kann die Gefährdung nicht beseitigt werden, sind diese Grundstücke nicht als Bauland geeignet und sollten in Grünland rückgewidmet werden. Auch für diese Entscheidungen ist eine entsprechende Koordination der örtlichen Raumplanung mit dem Wasserbau unerlässlich.

\subsection{Bereiche mit niedriger \\ Hochwassergefährdung - die Rolle der Bebauungsplanung}

Für Gebiete mit niedriger Hochwassergefährdung sieht Empfehlung 6 der ÖREK-Partnerschaft „Risikomanage- ment Hochwasser" ein Berücksichtigungsgebot für die örtliche Raumplanung vor, welches dem Prinzip der Risikovermeidung entspricht. Für Restrisikobereiche im Sinne von Überlastund Versagensfällen sieht Empfehlung 7 vor, Grundlagen zur Berücksichtigung des Restrisikos zu erarbeiten und Handlungsempfehlungen für Raumordnung und Baurecht abzuleiten (ÖROK 2018).

Für Gebiete mit niedriger Hochwassergefährdung und für Restrisikobereiche sehen die Raumordnungsgesetze der Bundesländer gegenwärtig nur vereinzelt Einschränkungen bei der Baulandwidmung vor: Das Niederösterreichische Raumordnungsgesetz kennt ein Baulandwidmungsverbot für BaulandSondergebiet mit Gefahrenpotenzial, Bauland-Industriegebiet und BaulandVerkehrsbeschränktes Industriegebiet im Abflussbereich des 300-jährlichen Hochwassers ( $\$ 15$ Abs 6 NÖROG), das Oberösterreichische Raumordnungsgesetz legt mit Bezug zum Restrisiko ein Widmungsverbot für Bauland in ehemals roten Zonen fest $(\$ 21$ Abs $1 \mathrm{a}$ OöROG). Ansonsten liegen Entscheidungen über die bauliche Nutzung von Räumen mit niedriger Hochwassergefährdung und Restrisiko im Planungsermessen der Gemeinden. Gefahreninformationen sind für diese Bereiche vorhanden bzw. werden die wasserbaulichen Fachdienste im Raumordnungsverfahren auf die bestehende Gefähr- 


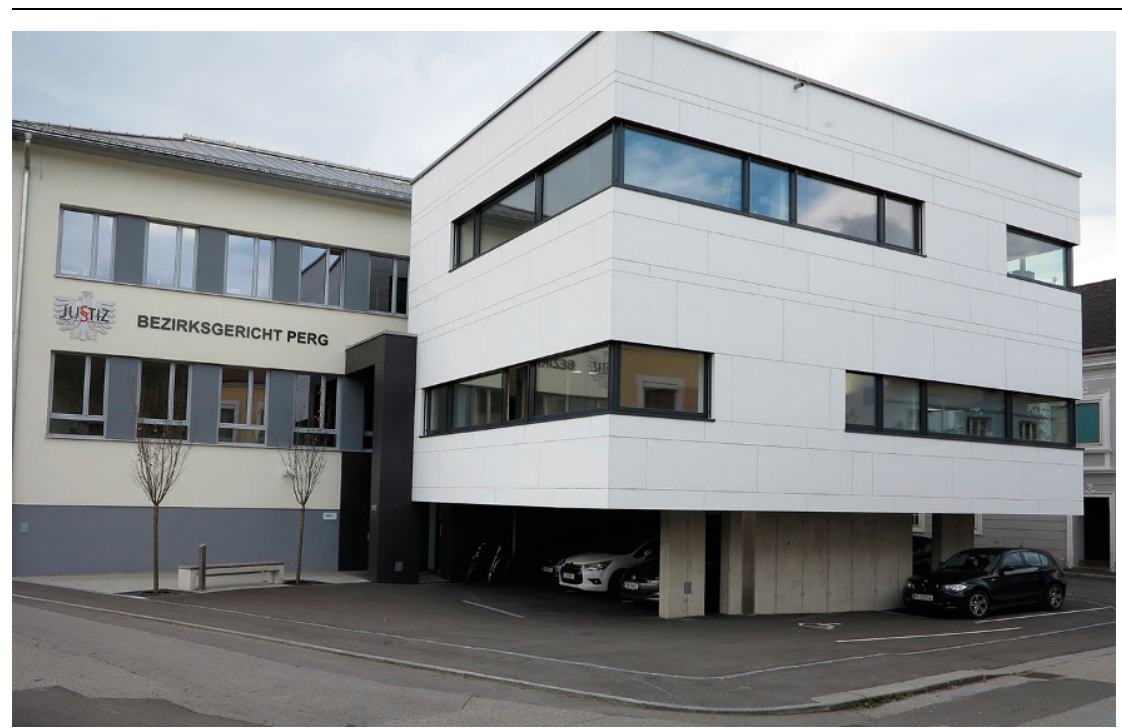

Abb. 3 Anpassung an Hochwassergefahren durch Erdgeschossnutzung als Parkplatz (Foto: W. Seher)

dung hinweisen. Zudem werden diese Gefährdungen im Zusammenhang mit der Auflage von Gefahrenzonenplänen kommuniziert. Die diesbezüglichen Widmungs- und Nutzungsentscheidungen der Gemeinden erfolgen daher nicht unter Unwissen. Die entstehenden Risiken werden bewusst in Kauf genommen (Fellinger 2020).

Eine Berücksichtigung von Gebieten mit niedriger Hochwassergefährdung und Restrisiko in der örtlichen Raumplanung umfasst die Information über die Gefährdung durch Ersichtlichmachung in den Planungsinstrumenten, Auflagen im Sinne einer hochwasserangepassten Bauweise im Flächenwidmungs-, aber besonders im Bebauungsplan, sowie die Festlegung von Widmungseinschränkungen in diesen $\mathrm{Ge}$ bieten. Letzteres erscheint aufgrund des beschränkten Dauersiedlungsraums in den alpinen Bundesländern nur sehr eingeschränkt umsetzbar, weshalb die folgenden Ausführungen die Berücksichtigung von Hochwassergefahren im Bebauungsplan zum Inhalt haben.

Hochwasserangepasste Bauformen können in der Bebauungsplanung verbindlich festgelegt werden. Für Neuerschließungen ermöglicht dieses Instrument der örtlichen Raumplanung Mindestvorgaben für Bauvorhaben in Gefährdungsbereichen im Sinne einer gefahrenangepassten Differenzierung der Nutzungsintensität und der Umsetzung einer baulichen Hochwasseranpassung (Abb. 3). Die Vorteile der Bebauungsplanung gegenüber gleichliegen in gebietsweisen einheitlichen Festlegungen, einer vorzeitigen Einbindung der Bauwerber sowie in der Bewusstseinsbildung für Hochwasserrisiken im Fall partizipativ gestalteter Planungsprozesse. Insbesondere Schäden aus pluvialen Hochwasserereignissen können oft durch einfache bauliche Maßnahmen vermieden werden. Die Freihaltung von Abflussgassen sowie Konzepte der Oberflächenentwässerung von zusammenhängenden Gebieten im Allgemeinen sind übliche Inhalte von Bebauungsplänen. Ferner können u.a. die Anordnung des Gebäudes am Grundstück, Bestimmungen zur Vorschreibung oder Untersagung von Geländeveränderungen, Flutmulden und Ableitungsbauwerke, wie Mauern und Dämme, in einem Bebauungsplan verankert werden. Eine enge $\mathrm{Ab}$ stimmung zwischen den Ortsplanern, den Gemeinden und dem Wasserbau bauungsplänen, die die Anpassung an Hochwassergefahren zum Inhalt haben, was Auswahl und Gestaltung der Maßnahmen betrifft, unerlässlich. Unterstützung bieten hier auch Informationsbroschüren zum hochwasserangepassten Bauen (vgl. z.B. ÖWAV 2013; BMNT 2019).

Die Berücksichtigung von Hochwassergefahren im Bebauungsplan ist $\mathrm{zu}$ forcieren. Die Bebauungsplanung wird besonders in ländlich geprägten Gemeinden selten angewendet, und wenn liegen Bebauungspläne oft lautenden Auflagen im Bauverfahren ist auch bei der Erstellung von Be- nur in Textform vor. Zudem können die Raumplanungsabteilungen in den Landesregierungen und der Wasserbau aufgrund nicht dokumentierter Kollaudierungen (Meldung der Baufertigstellung) oft nicht überprüfen, ob Festlegungen im Bebauungsplan dann tatsächlich im Bauverfahren umgesetzt werden. Hier ist auch die Koordination zwischen diesen Behörden und den Gemeinden als Baubehörden zu verbessern. Erlässt die Gemeinde keinen Bebauungsplan, beschränkt sich der Einfluss des Wasserbaus auf das Widmungsverfahren. Die Baulandwidmung legt aber nur in groben Zügen fest, wie eine Fläche genutzt wird und erlaubt in der Regel keine Anpassungsmaßnahmen an Hochwassergefahren. Ein kombinierter Flächenwidmungs- und Bebauungsplan, der in einigen Städten angewendet wird, würde konkretere Auflagen bezüglich Hochwasseranpassung bereits im Zuge der Widmung möglich machen.

\section{Resümee}

Der in der EU-Hochwasserrichtlinie zum Ausdruck kommende Paradigmenwechsel von der Gefahrenabwehr hin zum Risikomanagement hat die Anzahl der beteiligten Akteure im Hochwasserrisikomanagement erhöht und $\mathrm{zu}$ einem Bedeutungsgewinn flächenund raumbezogener Aspekte im Umgang mit Hochwassergefahren geführt (Löschner 2018; Hartmann und Jüpner 2014). Die Bedeutung von Fläche und Raum geht einher mit einem Bedarf nach intersektoraler Abstimmung zwischen den Akteuren im Hochwasserrisikomanagement, insbesondere zwischen der Raumplanung und dem Wasserbau. Die ÖREK-Partnerschaft „Risikomanagement Hochwasser" hat diesen Abstimmungsbedarf aufgegriffen und gibt mit den ÖROK-Empfehlungen Nr. 57 „Hochwasserrisikomanagement“ als Ergebnis einer inhaltlichen Koordination Leitlinien für den Umgang mit Hochwassergefahren und Hochwasserrisiken in der Raumplanung und für die administrative Koordination von Raumplanung und Wasserbau vor.

In diesem Beitrag wurde der Schwerpunkt auf jene Empfehlungen gelegt, die direkt auf die Koordination der überörtlichen bzw. örtlichen Raumplanung mit dem Wasserbau abzielen. Die in den ÖROK-Empfehlungen geforderte Berücksichtigung von Hochwasserabfluss- und Hochwasserrückhalteflächen 
in den Instrumenten der überörtlichen Raumplanung und hier besonders der Regionalplanung ist zu forcieren. Dabei ist einerseits die inhaltliche Koordination gefordert, die Freihaltung von Hochwasserabfluss- und Hochwasserrückhalteflächen als Anliegen der Regionalplanung in den Raumordnungsgesetzen zu verankern. Andererseits gilt es, auf Good-Practice-Beispielen wie der Blauzone Rheintal aufzubauen und im Wege der administrativen Koordination die Ausweisung von hochwasserbezogenen Vorrang- oder Freihaltezonen in regionalen Raumordnungsprogrammen sicherzustellen.

Die Berücksichtigung von Gebieten mit Überflutung hoher und mittlerer Wahrscheinlichkeit in der örtlichen Raumplanung zeigt sich in hohem Maße standardisiert. Diese Standardisierung beruht einerseits auf Widmungsbeschränkungen, die an Grenzwerte der Gefährdung gekoppelt und in den Raumordnungsgesetzen bzw. in Instrumenten der überörtlichen Raumplanung festgelegt sind. Nicht selten gehen diese Widmungsbeschränkungen auf eine inhaltliche Koordination von Raumplanung und Wasserbau zurück (vgl. z. B. die Entstehung und die aktuelle Überarbeitung des Sachprogramms Hochwasserschutz in der Steiermark). Anderseits gewährleisten Regeln der administrativen Koordination zwischen den Gemeinden und den wasserbaulichen Fachdiensten im Raumordnungsverfahren eine standardisierte Vorgehensweise bei der Gefahrenbeurteilung und den daraus folgenden Widmungsentscheidungen. Im Umgang mit gewidmetem Bauland in Zonen mit mittlerer Überflutungswahrscheinlichkeit und bei pluvialen Hochwassergefahren nimmt die administrative Koordination zwischen Raumplanung und Wasserbau einen hohen Stellenwert ein, da zwischen dem Raumplanungsziel einer Verdichtung der Siedlungsbereiche und dem Freihalten von Hochwasserabflussflächen abzuwägen ist und diese Abwägung entsprechende fachliche Grundlagen erfordert. Für Hochwasserabflussbereiche mit geringer Eintrittswahrscheinlichkeit und Restrisikogebiete gibt es im Raumordnungsrecht nur sehr vereinzelt normative Vorgaben. Die in den ÖROK-Empfehlungen angesprochene Berücksichtigung dieser Bereiche im Sinne einer Risikovermeidung wird daher überwiegend über informelle, nicht regulative Ansätze, wie Informationen, Bewusstseinsbildung oder anreizorientierte Systeme erfolgen, für die eine administrative Koordination von Raumplanung und Wasserbau aber ebenfalls von Bedeutung ist.

Im Umgang mit Hochwassergefahren und Hochwasserrisiken in der Raumplanung machen insbesondere Herausforderungen wie die Flächeninanspruchnahme für Bauland und Verkehrsflächen und der Klimawandel deutlich, dass im Hochwasserrisikomanagement eine interdisziplinäre, koordinierte Herangehensweise notwendig ist, um neue Risiken zu vermeiden und bestehende Risiken zu reduzieren. Die Raumplanung deckt mit der präventiven Vermeidung neuer Risiken einen wichtigen Bereich im Hochwasserrisikomanagement ab, in dem die notwendige Abstimmung mit dem Wasserbau auch über formale Vorgaben hinaus weiter intensiviert werden sollte.

Danksagung Teile des vorliegenden
Beitrags basieren auf Resultaten des Forschungsprojekts „PoCo-FLOOD“, das von der Österreichischen Akademie der Wissenschaften (ÖAW) im Rahmen des Forschungsprogramms „Earth System Sciences (ESS)“ gefördert wird.

Funding Open access funding provided by University of Natural Resources and Life Sciences Vienna (BOKU).

Open Access Dieser Artikel wird unter der Creative Commons Namensnennung 4.0 International Lizenz veröffentlicht, welche die Nutzung, Vervielfältigung, Bearbeitung, Verbreitung und Wiedergabe in jeglichem Medium und Format erlaubt, sofern Sie den/die ursprünglichen Autor(en) und die Quelle ordnungsgemäß nennen, einen Link zur Creative Commons Lizenz beifügen und angeben, ob Änderungen vorgenommen wurden.

Die in diesem Artikel enthaltenen Bilder und sonstiges Drittmaterial unterliegen ebenfalls der genannten Creative Commons Lizenz, sofern sich aus der Abbildungslegende nichts anderes ergibt. Sofern das betreffende Material nicht unter der genannten Creative Commons Lizenz steht und die betreffende Handlung nicht nach gesetzlichen Vorschriften erlaubt ist, ist für die oben aufgeführten Weiterverwendungen des Materials die Einwilligung des jeweiligen Rechteinhabers einzuholen.

Weitere Details zur Lizenz entnehmen Sie bitte der Lizenzinformation auf http://creativecommons.org/licenses/ by/4.0/deed.de.
Amt der Niederösterreichischen Landesregierung (o. J.): NÖ Hochwasserschutzzonenpläne. Online: https://www.noe.gv.at/noe/ Wasser/NOe_Hochwasserschutzzonenplaene. html, 11.11.2021.

Amt der Vorarlberger Landesregierung (2014): Blauzone: Regierungsbeschluss vom 17.12.2013. Online: https://presse.vorarlberg.at/land/servlet/ AttachmentServlet?action=show\&id=21492, 07.12.2021.

Amt der Vorarlberger Landesregierung (2013): Blauzone Rheintal. Verordnung der Vorarlberger Landesregierung über die Festlegung von überörtlichen Freiflächen zum Schutz vor Hochwasser im Rheintal. Bericht für die Landesregierung. Bregenz.

Bundesministerium für Land- und Forstwirtschaft, Umwelt und Wasserwirtschaft (BMLFUW) (Hrsg.) (2016): Leitfaden Gewäs- serentwicklung- und Risikomanagementkonzepte (GE-RM). Vorläufige Fassung 2017. Wien: Bundesministerium für Land- und Forstwirtschaft, Umwelt und Wasserwirtschaft. Online: https://info.bmlrt.gv.at/dam/jcr:5cb286e33975-454a-9611-4a15c1cb814c/Leitfaden_GERM_Vorlaeufige\%20Fassung_2017_final_ barrierefrei.pdf, 11.11.2021

Bundesministerium für Landwirtschaft, Regionen und Tourismus (BMLRT) (2021): 2. Nationaler Hochwasserrisikomanagementplan. Entwurf für die Öffentlichkeitsbeteiligung. Online: https://info.bmlrtgvat/dam/ jcr:0bda6510-d105-4aa4-843c-40419e5014a3/ RMP2021_Entwurf_barrierefrei_FINAL_online. pdf, 11.11.2021

Bundesministerium für Nachhaltigkeit und Tourismus (BMNT) (2019): Eigenvorsorge bei Oberflächenwasserabfluss. Ein Leitfaden für Pla- nung, Neubau und Anpassung. Online: https:// info.bmlrt.gv.at/dam/jcr:0416dad1-53f1-4816aab9-elb7d58bd66a/02\%20Brosch\%C3\%BCre Oberfl\%C3\%A4chenabfluss_BF_20190314.pdf, 11.11.2021

Dallhammer, E. (2006): Flächen sparen - Nachfrage lenken. In: Forum Land (Hrsg.): Flächen effizient nutzen. Raumordnung auf dem Prüfstand, 18-20. Eigenverlag, Wien.

Elmer, E., Hoymann, J., Düthmann, D., Vorogushyn, S., Kreibich, H. (2012): Drivers of flood risk in residential areas. Natural Hazards and Earth System Sciences 12:1641-1657. https:// doi.org/10.5194/nhess-12-1641-2012

Fellinger, R. (2020): Revision von Gefahrenzonenplänen und ihre Wirkung auf die örtliche Raumplanung. Die Wechselwirkung zwischen technischen Schutzmaßnahmen, Gefahrenzonen und räumlicher Entwicklung sowie der 
raumplanerische Umgang mit Restrisiken und Revisionsflächen in alpinen Regionen am Beispiel Vorarlberg. Masterarbeit am Institut für Raumplanung, Umweltplanung und Bodenordnung, Universität für Bodenkultur Wien.

Hartmann, T., Jüpner R. (2014): The Flood Risk Management Plan: An essential step towards the institutionalization of a paradigm shift. International Journal of Water Governance 2:107-118. https://doi.org/10.7564/13-IJWG5

Heiland, P. (2002): Vorsorgender Hochwasserschutz durch Raumordnung, interregionale Kooperation und ökonomischer Lastenausgleich. Verein zur Förderung des Institutes WAR, Darmstadt.

Kanonier, A. (2005): Naturgefahren im Österreichischen Raumordnungsrecht: In: Österreichische Raumordnungskonferenz (ÖROK) (2005): Präventiver Umgang mit Naturgefahren in der Raumordnung, Materialienband, 81-114. ÖROK Schriftenreihe Nr. 168, Wien.

Löschner, L., Herrnegger, M., Apperl, B., Senoner, T., Seher, W., Nachtnebel, H.P. (2017): Flood risk, climate change and settlement development: a micro-scale assessment of Austrian municipalities. Regional Environmental Change 17:311-322. https://doi.org/10.1007/s10113016-1009-0

Löschner, L. (2018): The spatial turn in flood risk management. A case study of Austria's changing flood policies. Dissertation am Institut für Raumplanung, Umweltplanung und Bodenordnung, Universität für Bodenkultur Wien.

Nachtnebel, H.P., Apperl, B. (2015): Beurteilung des Hochwasser-Schadenspotenzials unter dynamischen Bedingungen. Österreichische Wasser- und Abfallwirtschaft 67(3-4):120-130 https://doi.org/10.1007/s00506-015-0220-4

Nordbeck, R. (2014): Klimawandel und vorsorgender Hochwasserschutz in Österreich: eine entwicklungsdynamische Analyse der Anpassungskapazitäten (2002-2012). InFER Discussion Paper 2-2014, Wien.

Österreichische Raumordnungskonferenz (ÖROK) (2018): ÖROK-Empfehlung Nr. 57:
"Hochwasserrisikomanagement". Ausgangslage \& Rahmen, Empfehlungen, Erläuterungen \& Beispiele. Online: https://www.oerok gv.at/fileadmin/bestellservice/publikationen

pdf/broschuere_oerok-Empfehlung_Nr_57

Hochwasserrisikomanagement kurzfassungDE pdf, 11.11.2021

Österreichische Raumordnungskonferenz (ÖROK) (2021b): Anteil der Baulandreserven am Bauland insgesamt 2020. ÖROK-Atlas. Online: https://www.oerok-atlas.at/\#indicator/70 11.11.2021

Österreichische Raumordnungskonferenz (ÖROK) (2021a): 16. Raumordnungsbericht Analysen und Berichte zur räumlichen Entwicklung Österreichs 2018-2020. Eigenverlag, Wien. Österreichischer Wasser- und Abfallwirtschaftsverband (ÖWAV) (2013): Wassergefahren für Gebäude und Schutzmaßnahmen. ÖWAV-Leitfaden. Online:https://www.oewav.at/Publikationen? current $=293908 \&$ mode $=$ form, 11.11 .2021

Schmid, F., Neuhold, C., Wenk, M. (2022): Die Gefahrenzonenplanung als Fachgrundlage zum integralen Hochwasserrisikomanagement. Österreichische Wasser- und Abfallwirtschaft 74(3-4), in diesem Heft.

Seher, W. (2015): Potenziale der Grundzusammenlegung als Instrument des Flächenmanagements in ländlichen Räumen Österreichs. ZfV - Zeitschrift für Geodaesie, Geoinformation und Landmanagement, 140:365-372. DOI 10.12902/zfv-0082-2015

Seher, W. (2020a): Naturgefahrenrisiken in der Raumplanung. In: Stöglehner, G. (Hrsg.), Grundlagen der Raumplanung 2 - Strategien, Schwerpunkte, Konzepte, 179-199. Facultas Universitätsverlag, Wien.

Seher, W. (2020b): Mitigating Pluvial Flood Risks in Austria - The Contribution of Spatial Planning. In: Hepperle, E.; Paulsson, J.; Maliene V.; Mansberger, R.; Auzins, A.; Valciukiene, J. (Eds.), Methods and Concepts of Land Management - Diversity, Changes and New Approaches, 247-258; vdf Hochschulverlag AG an der ETH Zürich, Zürich.
Seher, W., Beutl, H. (2004): Möglichkeiten der Flächenvorsorge in Hochwasserabflussräumen Handlungsspielräume von Gemeinden und Gemeindekooperationen, am Beispiel von Gemeinden im Oberlauf der Triesting/Niederösterreich Studie im Rahmen von FloodRisk: Analyse de Hochwasserereignisse vom August 2002. Wien. Seher, W., Löschner, L. (2018): Instrumente der Raumplanung für die Flächenvorsorge gegenüber Hochwassergefahren. In: Kanonier, A., Rudolf-Miklau, F. (Hrsg.), Regionale Risiko Governance: Recht, Politik und Praxis, 445-459; Verlag Österreich, Wien.

Trein, P., Maggetti, M., Meyer, I. (2021): Necessary conditions for policy integration and administrative coordination reforms: an exploratory analysis, Journal of European Public Policy, 28(9):1410-1431. https://doi.org/10.1080/ 13501763.2020.1788121

Umweltbundesamt (2021): Flächeninanspruchnahme. Online: https://www.umweltbundesamt. at/umweltthemen/boden/

flaecheninanspruchnahme, 11.11.2021

Vogl, C. (2022): Rechtliche Möglichkeiten der wasserwirtschaftlichen Planung. Österreichische Wasser- und Abfallwirtschaft 74(3-4), in diesem Heft.

Warner, J. F., van Buuren, A., Edelenbos, J. (2012): Making space for the river. IWA Publishing.

Wenk, M., Mühlmann, H. (2022): Das Gewässerentwicklungs- und Risikomanagementkonzept Hintergründe und Erfahrungen aus dem Projekt LIFE IP IRIS. Österreichische Wasser- und Abfallwirtschaft 74(3-4), in diesem Heft.

Hinweis des Verlags Der Verlag bleibt in Hinblick auf geografische Zuordnungen und Gebietsbezeichnungen in veröffentlichten Karten und Institutsadressen neutral. 